\title{
Polyunsaturated fatty acid status and risk of type 1 diabetes in infants and children: a systematic review and meta-analysis
}

\author{
Xingmu Wang ${ }^{1}$, Shuping Zhong ${ }^{2}$, Jingfen Dong ${ }^{3}$, Fuyuan Zhuge ${ }^{3}$
}

\author{
${ }^{1}$ Clinical Laboratory Center, Shaoxing People's Hospital, Shaoxing Hospital of Zhejiang \\ University, Shaoxing, Zhejiang, China \\ 2Department of Hospital Management, Shaoxing People's Hospital, \\ Shaoxing Hospital of Zhejiang University, Shaoxing, Zhejiang, China \\ ${ }^{3}$ Department of Endocrine and Metabolism, Shaoxing People's Hospital, \\ Shaoxing Hospital of Zhejiang University, Shaoxing, Zhejiang, China
}

Submitted: 2 August 2019

Accepted: 18 February 2020

Arch Med Sci

DOI: https://doi.org/10.5114/aoms.2020.101299

Copyright $\odot 2020$ Termedia \& Banach

\section{Abstract}

Introduction: The existing findings about the association between polyunsaturated fatty acid (PUFA) status (especially long-chain n-3 PUFAs) and the risk of preclinical or clinical type 1 diabetes (T1D) in children are controversial. This review aimed to evaluate the definite association.

Material and methods: Three databases were systematically viewed until July, 2019 to identify relevant articles, without language restriction. Any observational study or randomized controlled trial reporting the risk estimates of preclinical or clinical T1D for PUFA status in infants and children was enrolled. Regardless of the statistical heterogeneity assessed by the I ${ }^{2}$ statistic, we pooled the odds ratios (ORs), relative risks (RRs) or hazard ratios (HRs) with 95\% confidence intervals $(\mathrm{Cl})$ through random-effects models.

Results: Five observational studies were enrolled in the meta-analysis. The status of $n-3$ PUFAs was negatively and significantly associated with the risk of preclinical, but not clinical, T1D (pooled RR $=0.85 ; 95 \% \mathrm{Cl}: 0.73-0.99$ ) with substantial heterogeneity $\left(I^{2}=72.2 \%\right)$. However, no such association was found between n-6 PUFA status and the risk of preclinical or clinical T1D.

Conclusions: The meta-analysis suggests that n-3 PUFA might play a potential protective role in the cause of preclinical T1D, and n-3 PUFA intake may be beneficial, since the n-3 PUFA status was associated with a significant decrease in the risk of preclinical T1D in children. Nevertheless, more well-designed prospective studies are necessary to determine whether dietary or supplemental intake of specific n-3 PUFA alters the risk of preclinical T1D.

Key words: polyunsaturated fatty acid status, type 1 diabetes, islet autoimmunity, risk, systematic review, meta-analysis.

\section{Introduction}

Type 1 diabetes (T1D), a chronic autoimmune disease, commonly attacks children [1], and its global incidence has increased for the last 10 years [2]. Before the clinical phase of T1D manifested with hyperglycemia, a months- or years-long asymptomatic period is observed, during which $\beta$-cell autoantibodies and their antigens are found in the

\author{
Corresponding author: \\ Fuyuan Zhuge \\ Department of Endocrine and \\ Metabolism \\ Shaoxing People's Hospital \\ Shaoxing Hospital \\ of Zhejiang University \\ No. 568, Zhongxing North \\ Road \\ Yuecheng District, Shaoxing, \\ Zhejiang \\ 312000, P.R. China \\ Tel.: +86 575-88228888 \\ Fax: +86 575-88228888 \\ E-mail: ffyyzg120@126.com
}


bloodstream. Examining the risk factors of autoantibodies, which appear prior to clinical diabetes, would reveal critical clues concerning the early pathogenic incidents of autoimmunity and probably the causes of T1D. The cause for autoimmunity is still unknown, but it may involve both a genetic background [3] and environmental factors [4, 5]. Dietary factors, such as polyunsaturated fatty acids (PUFAs)[6] and vitamin D [7, 8], are critical in the causes of T1D and in the initiation of autoimmunity that results in the occurrence of clinical diseases.

The existing findings about the association between PUFA status (especially long-chain n-3 PUFAs) and the risk of preclinical or clinical T1D in children are controversial, with some studies reporting a negative association $[9,10]$ and other studies reporting a positive association $[6,11]$. The inconsistent results among the previous studies might be attributed to the various patient characteristics, types of PUFAs, and biomarkers of PUFA status. Different PUFA status biomarkers reflect intake, and metabolism of fatty acids differs (lasting from 4-6 weeks for erythrocyte membrane [12] to 1-2 weeks for serum [13]). A recent systematic review and meta-analysis [14] suggests that the risk of preclinical T1D might be reduced by $n-3$ PUFA intake only in early life. It is unclear whether the effect depends on the improvement of the status of n-3 PUFA since conflicting results about intake/status and the risk of disease were found in previous reviews $[15,16]$. PUFA intake also does not necessarily lead to an equivalent change or other change in the status of PUFA [17]. Compared with PUFA intake, PUFA status may provide important insights into the role of PUFA in the pathogenesis of preclinical or clinical T1D. Biological effects of dietary PUFA depend on their ability to be absorbed in the intestine and transported to the systemic circulation and target tissues (i.e., bioavailability), which could greatly differ among individuals [18]. As reported, the possible protective effect of $n-3$ PUFA on islet autoimmunity (IA) may be attributed to a complicated interaction between the intake and gene-regulated desaturation of fatty acids [10]. Thus, PUFA status depends on both the diet intake and PUFA metabolism. Meanwhile, Zhao et al. [19] drew the conclusion that vitamin E may play a role in the maintenance of the plasma n-3 PUFA profile in humans with consequent desirable health effects since they found there are statistical relationships between plasma fatty acid profile and vitamin E intake.

Given the considerations above, we systematically reviewed and meta-analyzed the observational studies (OSs) and randomized controlled trials (RCTs) that have evaluated the association between PUFA status and the risk of preclinical or clinical T1D in infants and children.

\section{Material and methods}

\section{Search scheme and selection criteria}

Embase, Wiley Cochrane Central Register of Controlled Trials, and PubMed were systematically reviewed to identify clinical trials or observational studies published before July 18, 2019 (date of last search) following relevant guidelines [20, 21]. The search string broadly included "Fatty Acids," "Omega-3," "Omega-6," "Docosahexaenoic Acids," "Eicosapentaenoic Acid," "Linolenic Acids," "Linoleic Acids," "pufa," "polyunsaturated*," "type 1 diabetes," "IDDM," "T1DM," "islet autoimmunity," or "autoimmunity." We scanned the reference lists of the reports found in our search to identify other potentially relevant studies. The searches were performed by X.M.W. and J.F.D. independently, and any conflict between them was modulated by F.Y.ZG. The authors were contacted for detailed data if necessary. No ethical approval was required here.

\section{Inclusion and exclusion criteria}

The inclusion criteria included the following: (I) OSs or RCTs that evaluated the association between PUFA status and the risk of preclinical T1D (including islet or $\beta$-cell autoimmunity) or clinical T1D in infants and children without restriction of follow-up duration (the case definitions of preclinical and clinical T1D were based on the criteria provided by individual studies) and (II) relative risks (RRs), hazard ratios (HRs) or odds ratios (ORs) that were available, or data that were provided for their estimation. The exclusion criteria were as follows: (I) PUFA status that was evaluated from pregnant mothers and (II) quasi-RCT, cross-controlled trial, case report, comment, editorial letter, or unpublished article.

\section{Data extraction and quality assessment}

Demographic data (e.g., age and sex), maternal educational level, breastfeeding duration, highrisk genotype in the study populations, family history of T1D, and PUFA status biomarker were all extracted in standardized forms. The quality of each article was scored according to the Newcastle-Ottawa Scale (NOS) by X.M.W. and J.F.D. independently, and any discrepancy between them was addressed by re-evaluating the original article with F.Y.ZG. A score $>6$ stars, with a total of 9 stars, indicates high quality of an article [22].

\section{Statistical analysis}

The primary endpoints were the risk of preclinical or clinical T1D in infants and children. The RRs, HRs or ORs with $95 \%$ confidence intervals (Cls) of dichotomous data were pooled. PUFA mea- 
surements in different age groups from the same study were merged together before being pooled with measurements from the other studies. Moreover, the outcomes that only reported the association between some individual PUFA status and the risk of T1D in the same article were also merged. Separate analyses of PUFAs were prespecified given the different inherent functions.

Heterogeneity among studies was classified as minimal ( $\left.\right|^{2}$ statistic < $\left.25 \%\right)$, moderate $(<50 \%)$, or substantial ( $\geq 50 \%$ ) [23]. The $\mathrm{Cl}$ of $\mathrm{I}^{2}$ was estimated using an iterative noncentral chi-squared distribution [24]. Due to a priori heterogeneity of OSs, subgroups were designed using the following: (1) PUFA status assessment methods (PUFA composition of erythrocyte films [Group 1], serum PUFA level [Group 2]), (2) study design (case-control study [Group 1], nested case-control study [Group 2]), (3) region (United States [Group 1], Finland [Group 2]), (4) mean age of PUFA measurement (children [Group 1], infants [Group 2]), and (5) cohort group (DAISY (Diabetes Autoimmunity Study in the Young) cohort [Group 1], DIPP (Type 1 Diabetes Prediction and Prevention) cohort [Group 2]). Sensitivity analyses were also performed to identify any measurable origin of heterogeneity.

Publication bias was tested using the visually interpreted asymmetry of funnel plots and Egger's regression asymmetry test (significance at $p<$ 0.10 ) [25] and then treated using the trim-and-fill method [26]. All tests were completed using Stata 14.0 (Stata College Station, TX, USA) with a $p$ value in a two-tailed test less than 0.05 indicating significance.

\section{Results}

\section{Study selection, characteristics, and quality assessment}

The initial searches returned 405 potential articles, of which 31 articles were reserved for further examination. One study using the total serum PUFA status from the pregnant mother [27] was excluded. No RCT was found in our search. Finally, 5 OSs involving 1,757 patients were selected, including two nested case-control studies within a prospective cohort $[6,28]$ and three case-control studies in a prospective cohort [9-11] (Figure 1). Table I summarizes the key information of the 5 OSs, with sample sizes ranging from 167 to 674 patients. All 5 studies reported the outcomes of PUFA measurements from different age groups. One study only reported some specific, rather than total, PUFA status [6]. Data about the risk of preclinical and clinical T1D were presented in four OSs $[6,9,10,28]$ and one OS [11], respectively. The definitions of preclinical T1D varied substantially among studies. The 5 OSs are all highly qual-

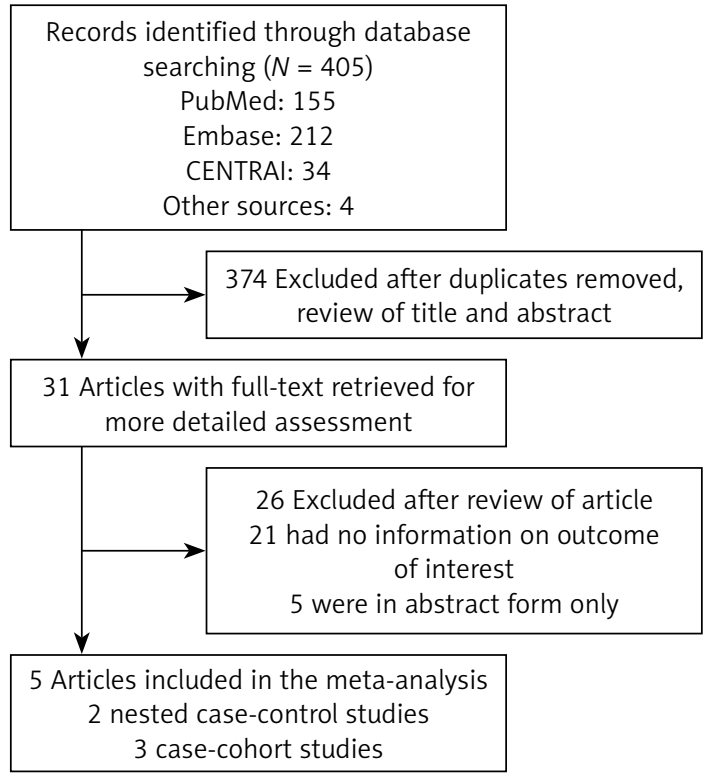

Figure 1. Flow chart of study selection

ified (NOS scores $\geq 7$, mean $=8.60$; Supplementary Table $\mathrm{SI})$.

Association between $n-3$ polyunsaturated fatty acid (PUFA) status and risk of preclinical or clinical type 1 diabetes (T1D)

In 4 OSs, n-3 PUFA status was significantly associated with a reduced risk of preclinical T1D in children (pooled $\mathrm{RR}=0.85 ; 95 \% \mathrm{Cl}$ : 0.73-0.99; $p=0.042)$, with substantial heterogeneity $\left(1^{2}=\right.$ $72.2 \%, p=0.013$; Figure 2). Similar results of $n-3$ PUFA status were observed in the Group 1 subgroup (pooled $\mathrm{RR}=0.57 ; 95 \% \mathrm{Cl}$ : 0.42-0.77; $p<$ 0.001 ), but not in the Group 2 subgroup ( $R R=$ 0.94; 95\% Cl: 0.89-1.00; $p=0.069$ ) (Supplementary Figure S1).

The evident publication bias ( $p=0.023$ in Egger's test) was processed by performing sensitivity analysis based on the trim-and-fill method. As a summary, potential negative unpublished articles were conservatively imputed to identify the positive articles that were responsible for the funnel plot asymmetry. To explore the publication bias based on the trim-and-fill method, we did not substitute the probable missing data and found basically similar results.

Only one study has suggested no association between n-3 PUFA status and the risk of clinical T1D in children [11].

\section{Association between n-6 PUFA status and risk of preclinical or clinical T1D}

The random effects model showed that $n-6$ PUFA status was not strongly associated with the risk of preclinical T1D in infants and children 


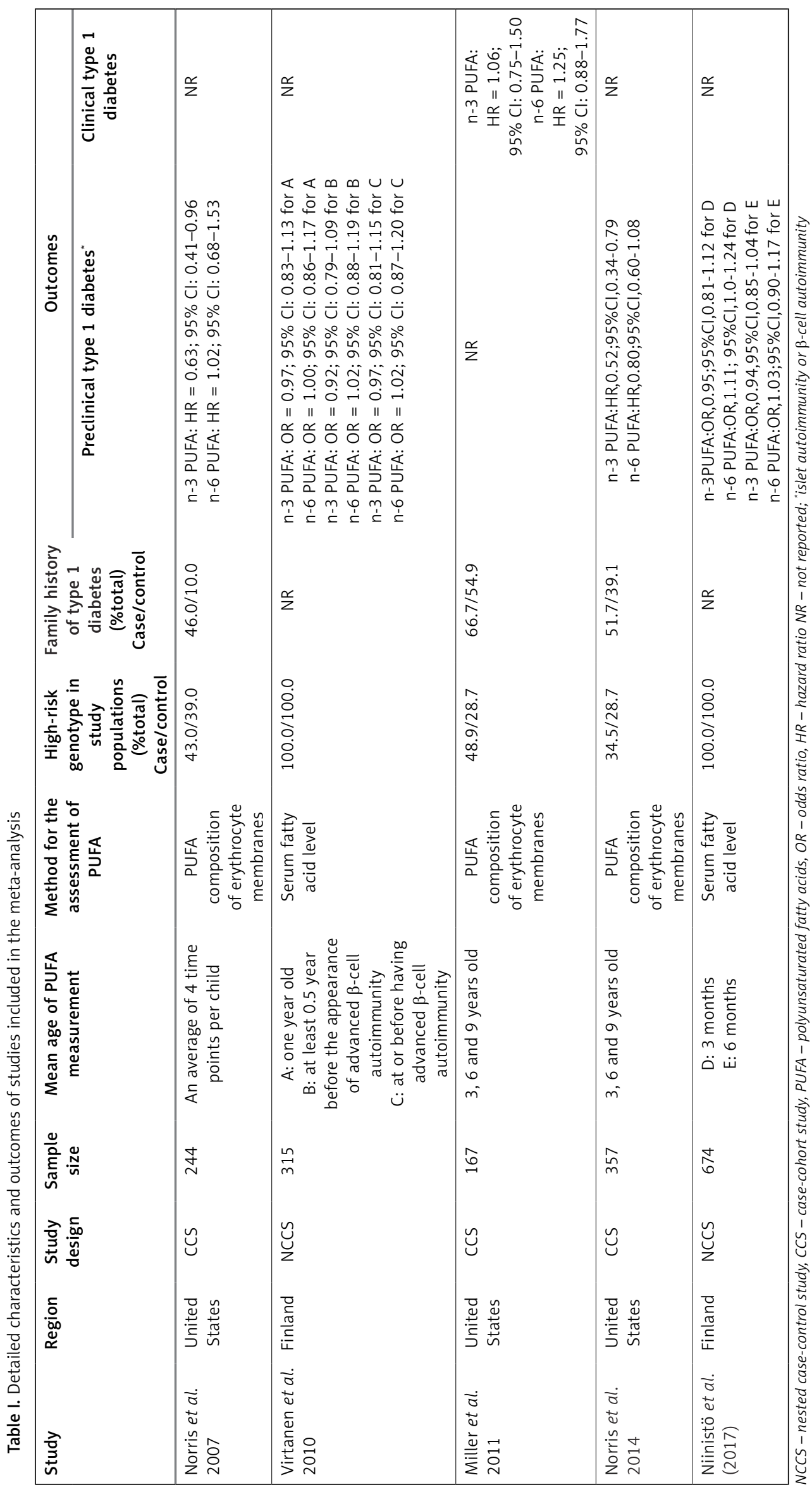




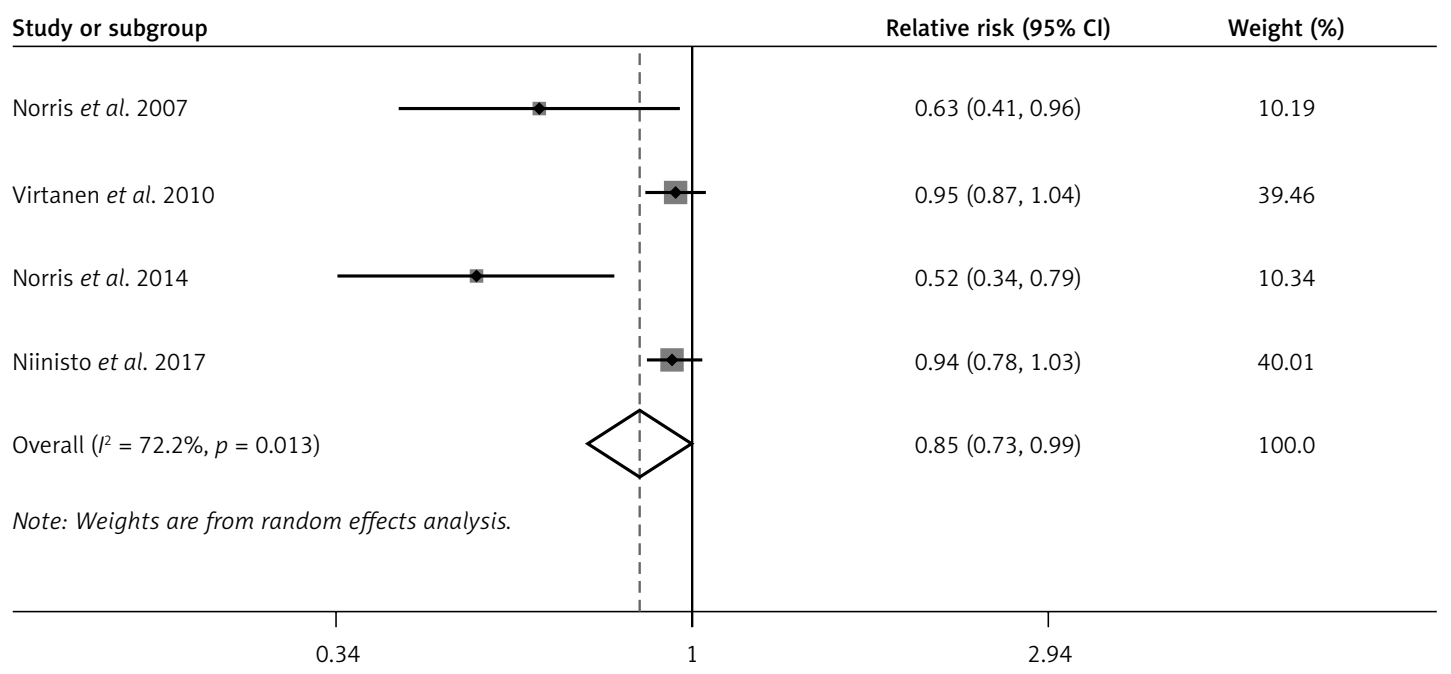

Figure 2. Forest plot of OSs on association between n-3 PUFA status and risk of preclinical T1D (X-axis: log scale; solid square: relative risk; horizontal lines: $95 \% \mathrm{Cls}$. The same in other figures)

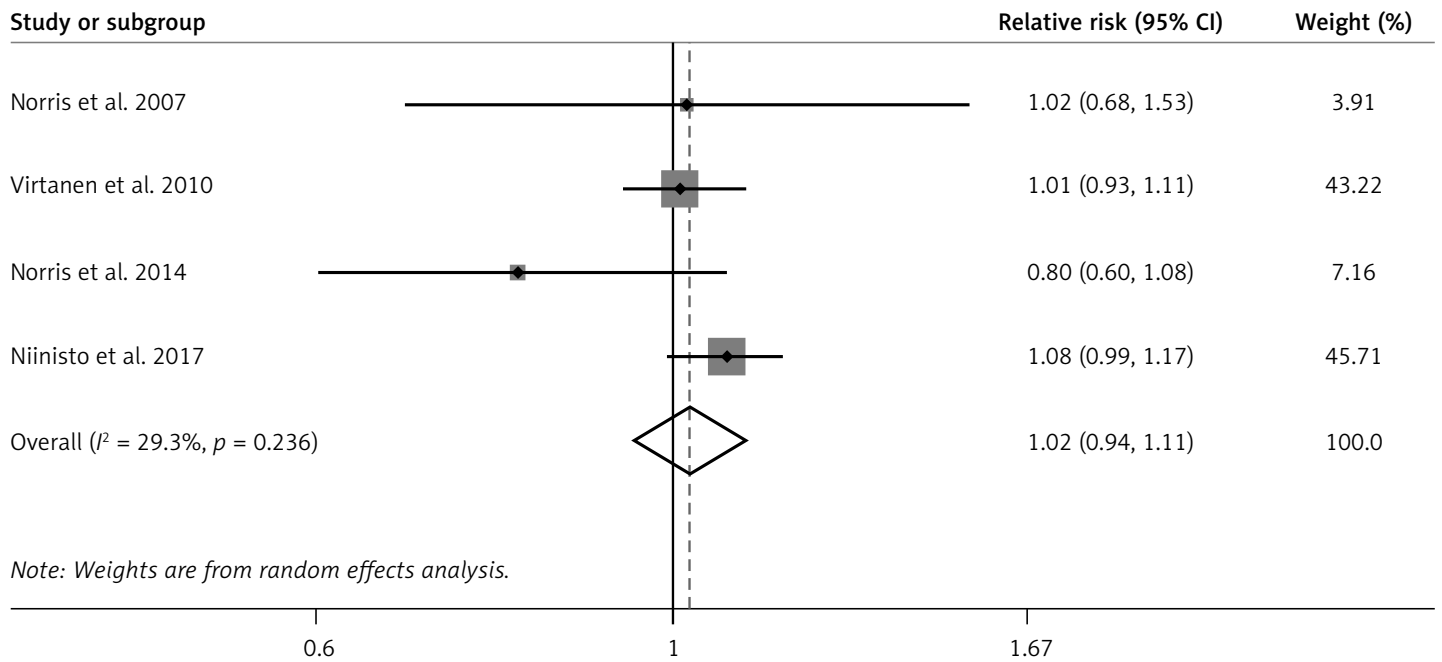

Figure 3. Forest plot of OSs on association between n-6 PUFA status and risk of preclinical T1D

(pooled RR $=1.02 ; 95 \% \mathrm{Cl}: 0.94-1.11 ; p=0.562$ ), with non-significant heterogeneity $\left(I^{2}=29.3 \%\right.$, $p=0.236$ ). Figure 3 shows the forest plots of this meta-analysis. Similar results were observed both in n-6 PUFA status in the Group 1 subgroup $(\mathrm{RR}=0.87 ; 95 \% \mathrm{Cl}: 0.69-1.10 ; p=0.251)$ and in n-6 PUFA status in the Group 2 subgroup (RR = 1.05; 95\% Cl: 0.98-1.02; $p=0.178$ ) (Supplementary Figure S2).

Neither Egger's test nor visual inspection revealed evident publication bias.

Moreover, no reliable association between $n-6$ PUFA status and the risk of clinical T1D was reported in one study [11].

\section{Discussion}

As far as we know, we were the first to systematically review and meta-analyze the association between PUFA status and the risk of preclinical or clinical T1D in infants and children. This work, searching all existing studies and involving 1,757 children, shows a significant association between n-3 PUFA status and the risk of preclinical T1D, but only 4 OSs reported the relevant RRs in infants and children. Two studies reported a negative association $[9,10]$, but the other two studies reported a positive association $[6,9,28]$. The between-study heterogeneity could be ascribed to the methodological differences in addition to genetic risk in the study populations. For instance, (1) the expression of preclinical T1D was diverse among studies. They shared a common expression, with positivity for insulin autoantibodies, glutamic acid decarboxylase antibodies (GADAs) or the $65 \mathrm{kDa}$ isoform of GADAs, or insulinoma-related antigen-2 autoantibodies. Some studies confirmed the presence of clinical T1D or the repeated positivity for islet cell autoantibodies besides common expression. (2) The mean ages of the subjects using fatty acid measurements were very distinct. In the first study, membrane fatty acid data were available for 4 time points 
per child on average in the 214 subcohort population [9]. In the second study, the serum fatty acids were measured 1 year, at least half year, before the subsequent occurrence of $\beta$-cell autoimmunity, and upon or before the transition to advanced $\beta$-cell autoimmunity [6]. Another study reported the $n-3$ and n-6 erythrocyte fatty acid contents in 3-, 6-, and 9-year-olds [10]. Moreover, serum fatty acids were reported in 3-and 6-month-old infants [28]. It was found that long-chain n-3 PUFAs could protect infants from the possible development of preclinical T1D during their infancy, in which the immune system is maturing and developing. We previously found that n-3 PUFA intake only in early life might reduce the risk of preclinical T1D [14] based on a subanalysis enrolling only one study [9]. However, it was incomprehensible since a significant difference was only found in n-3 PUFA status in the older age subgroup (DAISY cohort), but not in the younger age subgroup (DIPP cohort), in the present meta-analysis. One possibility is that PUFA intake in infancy or even in pregnancy plays a pivotal role in reducing the risk of preclinical T1D by increasing the status of n-3 PUFA in children or later in life. Liu et al. [14] only evaluated the effect of a total n-3 PUFA intake on the risk of preclinical T1D. This seems to suggest that only when the nutrient intake is insufficient may the nutrient supplementation have benefits. However, when dietary intake is adequate, additional intake from supplementation may not confer further benefit [29]. Another possibility is that IA also could be predicted by specific n-3 fatty acids (e.g., docosapentaenoic acid), but not by $\alpha$-linolenic acid, EPA, or DHA [10].

This meta-analysis shows no significant association between n-3 PUFA status and the risk of clinical T1D in infants and children. One study using the total serum PUFA status from the pregnant mother also showed no significant association between maternal serum EPA or DHA and risk of T1D in the offspring [27]. One probability is that the slight difference in n-3 PUFA status can affect the risk of the disease at early stage (i.e., transformation to IA), but is insufficient to impact the transition to diabetes in the case of a further disease process and more severe inflammation. We found that n-6 PUFA status was not associated with the risk of preclinical or clinical T1D since $n-6$ PUFAs may promote inflammation by inhibiting n-3 PUFA synthesis and by acting as precursors to proinflammatory eicosanoids, for the following reason: the $n-6: n-3$ PUFA ratio in the diet has a higher possibility of affecting the risk of T1D than n-6 PUFAs alone [30].

This review has several advantages, such as the extensive searches based on standard Cochrane protocols. However, it also has some lim- itations. First, RCTs or unpublished reports were not enrolled, and the relatively small sample sizes of the included studied might bias our results. Meanwhile, we pooled relative risk data (OR, HR, $\mathrm{RR}$ ) from different publications based on the same study, so that even though they are not identical, there is a large overlap in the participants. Second, significant heterogeneity for some outcomes was observed as expected. Third, no included study focused on the PUFA status of the pregnant mother since it was reported only in one study [27]. Fourth, the reason for the absence of an association between n-3 PUFA status and the risk of clinical T1D, with preclinical T1D showing an association, is not definitely elucidated because of the small number of available studies. Moreover, Egger's test evaluating the association between n-3 PUFA status and the risk of preclinical T1D shows potential publication bias, which might undermine the real effect if important articles were missed.

In summary, the meta-analysis suggests that n-3 PUFA might play a potential protective role in the cause of preclinical T1D, and n-3 PUFA intake may be beneficial, since the n-3 PUFA status was associated with a significant decrease in the risk of preclinical T1D in children. Nevertheless, more well-designed prospective studies are necessary to determine whether dietary or supplemental intake of specific n-3 PUFA alters the risk of preclinical T1D.

\section{Acknowledgments}

Xingmu Wang and Shuping Zhong contributed equally to this work.

\section{Conflict of interest}

The authors declare no conflicts of interest.

\section{References}

1. Katsarou A, Gudbjörnsdottir S, Rawshani A, et al. Type 1 diabetes mellitus. Nat Rev Dis Primers 2017; 3: 17016.

2. Tao Z, Shi A, Zhao J. Epidemiological perspectives of diabetes. Cell Biochem Biophys 2015; 73: 181-5.

3. Pociot F, Lernmark Å. Genetic risk factors for type 1 diabetes. Lancet 2016; 387: 2331-9.

4. Rewers M, Ludvigsson J. Environmental risk factors for type 1 diabetes. Lancet 2016; 387: 2340-8.

5. Butalia S, Kaplan GG, Khokhar B, Rabi DM. Environmental risk factors and type 1 diabetes: past, present, and future. Can J Diabetes 2016; 40: 586-93.

6. Virtanen SM, Niinistö S, Nevalainen J, et al. Serum fatty acids and risk of advanced beta-cell autoimmunity: a nested case-control study among children with HLA-conferred susceptibility to type I diabetes. Eur J Clin Nutr 2010; 64: 792-9.

7. Dong JY, Zhang WG, Chen JJ, Zhang ZL, Han SF, Qin LQ. Vitamin $D$ intake and risk of type 1 diabetes: a meta-analysis of observational studies. Nutrients 2013; 5: 3551-62. 
8. Ismail MM, Abdel Hamid TA, Ibrahim AA, Marzouk H. Serum adipokines and vitamin D levels in patients with type 1 diabetes mellitus. Arch Med Sci 2017; 13: 738-44.

9. Norris JM, Yin X, Lamb MM, et al. Omega-3 polyunsaturated fatty acid intake and islet autoimmunity in children at increased risk for type 1 diabetes. JAMA 2007; 298: $1420-8$.

10. Norris JM, Kroehl M, Fingerlin TE, et al. Erythrocyte membrane docosapentaenoic acid levels are associated with islet autoimmunity: the Diabetes Autoimmunity Study in the Young. Diabetologia 2014; 57: 295-304.

11. Miller MR, Yin X, Seifert J, et al. Erythrocyte membrane omega- 3 fatty acid levels and omega- 3 fatty acid intake are not associated with conversion to type 1 diabetes in children with islet autoimmunity: the Diabetes Autoimmunity Study in the Young (DAISY). Pediatr Diabetes 2011; 12: 669-75.

12. Baur LA, O'Connor J, Pan DA, Wu BJ, O’Connor MJ, Storlien LH. Relationships between the fatty acid composition of muscle and erythrocyte membrane phospholipid in young children and the effect of type of infant feeding. Lipids 2000; 35: 77-82.

13. Arab L. Biomarkers of fat and fatty acid intake. J Nutr 2003; 133 Suppl 3: 925S-932S.

14. Liu X, Zhang $\mathrm{Y}, \mathrm{Wu} \mathrm{H}$, et al. Intake of polyunsaturated fatty acids and risk of preclinical and clinical type $1 \mathrm{di}$ abetes in children-a systematic review and meta-analysis. Eur J Clin Nutr 2019; 73: 1-8.

15. Wei $H$, Jing $H$, Wei $Q$, Wei $G$, Heng Z. Associations of the risk of lung cancer with serum 25-hydroxyvitamin $D$ level and dietary vitamin D intake: a dose-response PRISMA meta-analysis. Medicine (Baltimore) 2018; 97 : e12282.

16. Moazzen S, Dolatkhah R, Tabrizi JS, et al. Folic acid intake and folate status and colorectal cancer risk: A systematic review and meta-analysis. Clin Nutr 2018; 37: 1926-34.

17. Ruiz-Núñez B, Kuipers RS, Luxwolda MF, et al. Saturated fatty acid (SFA) status and SFA intake exhibit different relations with serum total cholesterol and lipoprotein cholesterol: a mechanistic explanation centered around lifestyle-induced low-grade inflammation. J Nutr Biochem 2014; 25: 304-12.

18. Flachs P, Rossmeisl M, Kopecky J. The effect of n-3 fatty acids on glucose homeostasis and insulin sensitivity. Physiol Res 2014; 63 Suppl 1: S93-118.

19. Zhao Y, Monahan FJ, McNulty BA, et al. Plasma n-3 polyunsaturated fatty status and its relationship with vitamin E intake and plasma level. Eur J Nutr 2017; 56: 1281-91.

20. Moher D, Liberati A, Tetzlaff J, Altman DG. Preferred reporting items for systematic reviews and meta-analyses: the PRISMA statement. Ann Intern Med 2009; 151: 264-9, W64.

21. Postolica R, lorga M, Savin M, Azoicai D, Enea V. The utility of Leventhal's model in the analysis of the psycho-behavioral implications of familial cancer - a literature review. Arch Med Sci 2018; 14: 1144-54.

22. Kawalec P, Holko P, Gawin M, Pilc A. Effectiveness of fixed-dose combination therapy in hypertension: systematic review and meta-analysis. Arch Med Sci 2018; 14: 1125-36.

23. Rezaie A, Gu P, Kaplan GG, Pimentel M, Al-Darmaki AK. Dyssynergic defecation in inflammatory bowel disease: a systematic review and meta-analysis. Inflamm Bowel Dis 2018; 24: 1065-73.
24. Hedges LV, Pigott TD. The power of statistical tests in meta-analysis. Psychol Methods 2001; 6: 203-17.

25. Yang Y, Liu DC, Wang QM, et al. Alcohol consumption and risk of coronary artery disease: A dose-response meta-analysis of prospective studies. Nutrition 2016; 32: 637-44.

26. Duval S, Tweedie R. Trim and fill: a simple funnel-plotbased method of testing and adjusting for publication bias in meta-analysis. Biometrics 2000; 56: 455-63.

27. Sørensen IM, Joner G, Jenum PA, Eskild A, Stene LC. Serum long chain n-3 fatty acids (EPA and DHA) in the pregnant mother are independent of risk of type 1 diabetes in the offspring. Diabetes Metab Res Rev 2012; 28: 431-8.

28. Niinistö S, Takkinen HM, Erlund I, et al. Fatty acid status in infancy is associated with the risk of type 1 diabetes-associated autoimmunity. Diabetologia 2017; 60: 1223-33.

29. Fernández-Cao JC, Warthon-Medina M, Moran VH, et al. Zinc intake and status and risk of type 2 diabetes mellitus: a systematic review and meta-analysis. Nutrients 2019; 11: 1027.

30. Raheja BS, Sadikot SM, Phatak RB, Rao MB. Significance of the N-6/N-3 ratio for insulin action in diabetes. Ann N Y Acad Sci 1993; 683: 258-71. 